Page 1 of 6

Supporting Information for

Development of the ${ }^{99} \mathrm{~m}$ Tc-Hydroxamamide Complex as a Probe Targeting

\title{
Carbonic Anhydrase IX
}

Shimpei Iikuni, ${ }^{\dagger}$ Keiichi Tanimura, ${ }^{\dagger}$ Hiroyuki Watanabe, Yoichi Shimizu, Hideo Saji, and Masahiro Ono*

Department of Patho-Functional Bioanalysis, Graduate School of Pharmaceutical Sciences, Kyoto University, 46-29 Yoshida Shimoadachi-cho, Sakyo-ku, Kyoto 606-8501, Japan.

${ }^{\dagger}$ S.I. and K.T. contributed equally to this work.

Corresponding Author

*Phone: +81-75-753-4556; Fax: +81-75-753-4568; E-mail: ono@pharm.kyoto-u.ac.jp. 
Page 2 of 6

Table S1. Radiochemical Yield and Radiochemical Purity of $\left[{ }^{99 \mathrm{~m}} \mathrm{Tc}\right] \mathrm{Ham}$ and [99m Tc]MHam Complexes

\begin{tabular}{|c|c|c|}
\hline compound & radiochemical yield (\%) & radiochemical purity $(\%)$ \\
\hline$\left[{ }^{99 \mathrm{~m}} \mathrm{Tc}\right] \mathrm{SAB} 2 \mathrm{~A}$ & 14.4 & \\
\hline$\left[{ }^{99 \mathrm{~m}} \mathrm{Tc}\right] \mathrm{SAB} 2 \mathrm{~B}$ & 22.5 & \\
\hline$\left[{ }^{99 \mathrm{~m}} \mathrm{Tc}\right] \mathrm{URB} 2 \mathrm{~A}$ & 39.1 & \\
\hline \multirow[t]{2}{*}[{}^{99m}\mathrm{Tc}]{$\mathrm{URB} 2 \mathrm{~B}$} & 31.7 & \\
\hline & & $>95.0$ \\
\hline$\left[{ }^{99 \mathrm{~m}} \mathrm{Tc}\right] \mathrm{BHamA}$ & 38.8 & \\
\hline$\left[{ }^{99 \mathrm{~m}} \mathrm{Tc}\right] \mathrm{BHamB}$ & 30.9 & \\
\hline$\left[{ }^{99 \mathrm{~m}} \mathrm{Tc}\right] \mathrm{MURB} 2$ & 44.1 & \\
\hline$\left[{ }^{99 m} \mathrm{Tc}\right] \mathrm{MBHam}$ & 63.8 & \\
\hline
\end{tabular}


Table S2. Radioactivity of Extracted Organs after Intravenous Injection of ${ }^{\text {[99m }}$ Tc]URB2A in the HT-29 Tumor-Bearing Mice

\begin{tabular}{|c|c|c|c|c|c|}
\hline \multirow[b]{2}{*}{ organ } & \multicolumn{5}{|c|}{ time after injection } \\
\hline & $1 \mathrm{~h}$ & $3 \mathrm{~h}$ & $6 \mathrm{~h}$ & $24 \mathrm{~h}$ & $\begin{array}{l}\quad 24 \mathrm{~h}+ \\
\text { blocking* }\end{array}$ \\
\hline blood & $9.52 \pm 0.95$ & $5.40 \pm 0.96$ & $3.78 \pm 0.43$ & $2.07 \pm 0.26$ & $1.66 \pm 0.18^{\S}$ \\
\hline spleen & $10.09 \pm 1.36$ & $5.09 \pm 1.28$ & $2.47 \pm 0.38$ & $1.37 \pm 0.08$ & $1.19 \pm 0.13^{\S}$ \\
\hline pancreas & $3.59 \pm 0.32$ & $1.80 \pm 0.35$ & $0.90 \pm 0.25$ & $0.45 \pm 0.11$ & $0.49 \pm 0.09$ \\
\hline $\operatorname{stomach}^{\dagger}$ & $4.38 \pm 2.06$ & $2.74 \pm 0.74$ & $3.11 \pm 0.63$ & $0.42 \pm 0.07$ & $0.83 \pm 0.26^{\S}$ \\
\hline intestine & $8.63 \pm 1.36$ & $9.77 \pm 1.43$ & $4.52 \pm 1.77$ & $3.34 \pm 0.15$ & $3.49 \pm 0.84$ \\
\hline kidney & $32.82 \pm 6.75$ & $19.36 \pm 0.57$ & $13.56 \pm 1.41$ & $6.17 \pm 0.44$ & $4.12 \pm 0.63$ \\
\hline liver & $8.59 \pm 0.88$ & $6.23 \pm 0.61$ & $4.02 \pm 0.54$ & $2.60 \pm 0.22$ & $2.38 \pm 0.34$ \\
\hline heart & $7.25 \pm 0.77$ & $3.02 \pm 0.63$ & $2.16 \pm 0.41$ & $1.17 \pm 0.10$ & $1.09 \pm 0.23$ \\
\hline lung & $14.76 \pm 1.88$ & $7.88 \pm 1.70$ & $6.12 \pm 0.67$ & $3.15 \pm 0.42$ & $3.19 \pm 0.64$ \\
\hline tumor & $3.44 \pm 0.50$ & $3.13 \pm 0.32$ & $2.75 \pm 0.34$ & $2.18 \pm 0.10$ & $1.62 \pm 0.18$ \\
\hline muscle & $2.70 \pm 0.68$ & $1.29 \pm 0.32$ & $0.69 \pm 0.14$ & $0.44 \pm 0.08$ & $0.33 \pm 0.10$ \\
\hline
\end{tabular}


Page 4 of 6

brain $\quad 0.23 \pm 0.04 \quad 0.13 \pm 0.03 \quad 0.10 \pm 0.02 \quad 0.07 \pm 0.01 \quad 0.06 \pm 0.01$

Values are expressed as the \% injected dose per gram of organ. Each value is the mean \pm standard deviation of five mice. *Coinjection of acetazolamide (10 mg/kg mouse).

$\dagger$ Values are expressed as the \% injected dose. ${ }^{\S} \mathrm{P}<0.05$, and ${ }^{\ddagger} \mathrm{P}<0.001$ (Student's $t$-test). 
Table S3. Radioactivity of Extracted Organs after Intravenous Injection of $\left.{ }^{99 \mathrm{~m}} \mathrm{Tc}\right]$ MURB2 in the HT-29 Tumor-Bearing Mice

time after injection

\begin{tabular}{|c|c|c|c|c|c|}
\hline organ & $1 \mathrm{~h}$ & $3 \mathrm{~h}$ & $6 \mathrm{~h}$ & $24 \mathrm{~h}$ & $\begin{array}{l}24 \mathrm{~h}+ \\
\text { blocking* }\end{array}$ \\
\hline blood & $7.10 \pm 0.61$ & $4.94 \pm 0.87$ & $2.95 \pm 0.27$ & $0.81 \pm 0.33$ & $0.66 \pm 0.16$ \\
\hline spleen & $2.00 \pm 0.18$ & $1.47 \pm 0.14$ & $1.10 \pm 0.15$ & $0.33 \pm 0.12$ & $0.28 \pm 0.11$ \\
\hline pancreas & $2.27 \pm 0.39$ & $1.63 \pm 0.52$ & $0.93 \pm 0.19$ & $0.38 \pm 0.22$ & $0.37 \pm 0.09$ \\
\hline stomach $^{\dagger}$ & $19.49 \pm 4.62$ & $5.00 \pm 0.56$ & $4.05 \pm 0.62$ & $3.87 \pm 1.23$ & $3.98 \pm 0.58$ \\
\hline intestine & $4.00 \pm 0.59$ & $7.42 \pm 1.87$ & $11.22 \pm 1.69$ & $3.76 \pm 1.49$ & $4.51 \pm 1.38$ \\
\hline kidney & $5.09 \pm 0.50$ & $5.60 \pm 0.93$ & $7.62 \pm 1.41$ & $1.89 \pm 0.36$ & $1.45 \pm 0.28$ \\
\hline liver & $5.34 \pm 0.36$ & $4.57 \pm 0.58$ & $4.47 \pm 0.44$ & $1.45 \pm 0.25$ & $1.40 \pm 0.26$ \\
\hline heart & $1.86 \pm 0.28$ & $1.36 \pm 0.30$ & $0.84 \pm 0.11$ & $0.29 \pm 0.13$ & $0.21 \pm 0.11$ \\
\hline lung & $5.44 \pm 0.48$ & $4.32 \pm 0.93$ & $2.94 \pm 0.17$ & $0.80 \pm 0.24$ & $0.58 \pm 0.16$ \\
\hline tumor & $2.10 \pm 0.24$ & $1.87 \pm 0.22$ & $1.45 \pm 0.06$ & $0.73 \pm 0.18$ & $0.57 \pm 0.10$ \\
\hline muscle & $0.70 \pm 0.09$ & $0.52 \pm 0.13$ & $0.28 \pm 0.13$ & $0.15 \pm 0.12$ & $0.12 \pm 0.09$ \\
\hline
\end{tabular}


Page 6 of 6

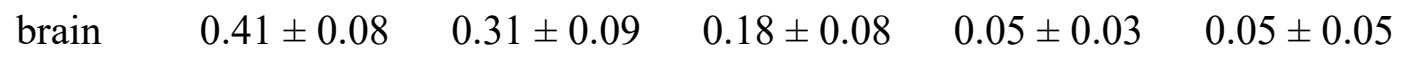

Values are expressed as the \% injected dose per gram of organ. Each value is the mean \pm standard deviation of five mice. *Coinjection of acetazolamide (10 mg/kg mouse).

†Values are expressed as the $\%$ injected dose. 